\title{
Maxillary and Palatal Fracture With Near-Avulsion of Bone Fragment-Case Report
}

\author{
Inês Insua-Pereira*, Pedro Ferreira, Joana Costa, Sérgio Teixeira and Álvaro Silva \\ Department of Plastic Reconstructive, Portugal
}

*Corresponding author: Inês Ínsua Pereira, Department of Plastic Reconstructive and Aesthetic Surgery and Burn Unit, Centro Hospitalar de São João, Porto, Portugal, Email: inesinsua@gmail.com

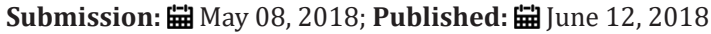

\begin{abstract}
Facial trauma can assume complex patterns closely related to the mechanisms of injury, often associating soft tissue injuries with bone fractures, sometimes with important functional and esthetic impact. The approach of such complex injuries must be individualized and basic principles of diagnosis, preoperative planning, wound care and postoperative management must be applied. We present a case of unusual orofacial trauma involving a complex fracture of the maxilla with associated soft tissue lacerations, involving the alveolar process, hard palate, maxillary sinus and nasal floor with near-avulsion of the fragment which was pediculated only by the anterior vestibular mucosa. The approach and surgical treatment of this case is presented along with a discussion of the management of this type of injuries.
\end{abstract}

\section{Case Report}

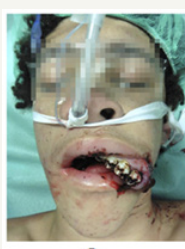

a

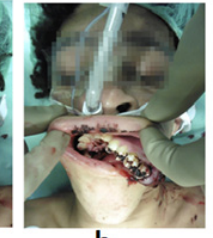

b

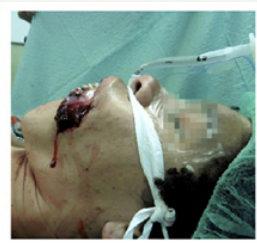

c

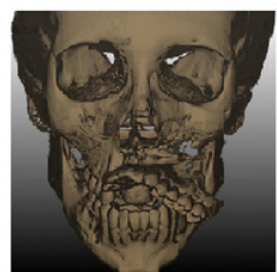

d

Figure 1: Initial presentation of patient. 1a-1c: Patient after sedation and intubation in the operating room. 1(d): Highresolution $\mathrm{CT}$ with $3 \mathrm{D}$ reconstruction.

The patient is a 16-year-old male that suffered an injury by running at high speed into a metal wire during football practice. The wire caused an intra-oral laceration in a postero-anterior direction, with an entry point in interdental spaces, causing a direct bone

fracture in the alveolar process and palate in an oblique pattern, as well as fracture of the anterior wall of the left maxillary sinus and nasal floor. This combination of injuries caused a dental-bearing disjuncted bone fragment of the maxilla pediculated only by the anterior vestibular mucosa (Figure 1a-1c). The patient was awake and alert on admission in the emergency department. Clinical examination confirmed a clear airway and no signs of associated lesions. His vital signs were stable and there was no active bleeding.
Poor oral hygiene and multiple dental caries were noted. The patient was submitted to a face high-resolution computed tomography (CT) with 3D reconstruction for better characterization of the visible fracture and exclusion of associated lesions (Figure 1d). The patient was submitted to emergent surgery for reduction of the bone fragment and microplate osteosynthesis by approach through existing lacerations. The osteosynthesis was performed in three locations: the anterior maxilla bilaterally through the lateral superior vestibular mucosa lacerations and the palate also trough the existing laceration (Figure 2a-2c). The upper dental arch was immobilized with Erich arch bar fixation and steel wire cerclage of dental pieces (Figure $2 \mathrm{~d}$ ). By the end of the procedure, a satisfactory reduction of the bone fragment was achieved without 
its vascular compromise. Oral antibiotic prophylaxis (amoxicillin plus clavulanate) and oral anti-inflammatory (ibuprofen) were administered and a liquid diet was prescribed in the first postoperative week.

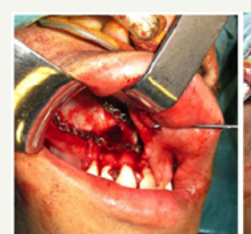

a
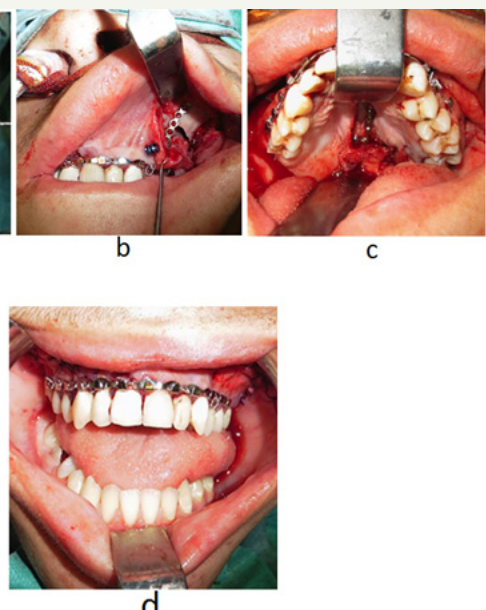

d
Figure 2: Surgical treatment of fracture a-c. Microplate osteosynthesis of the right anterior maxilla (a), left anterior maxila (b) and hard palate (c), Erich arch bar and steel wire immobilization of upper dental arch (d).

In the first postoperative day there was slight venous congestion of the most distal mucosal flaps of the fragment, with progressive improvement over the course of the next days. Approximately two weeks after surgery, a small dehiscence of the palatine suture was noted, that healed without the need for additional intervention. Erich arch bar was removed after two weeks of surgery and dental cerclage was removed after four weeks of surgery. Four months after the injury, there were no functional consequences and a good esthetic outcome was achieved, with restoration of previous dental occlusion, as confirmed by the patient (Figure 3a-3d).

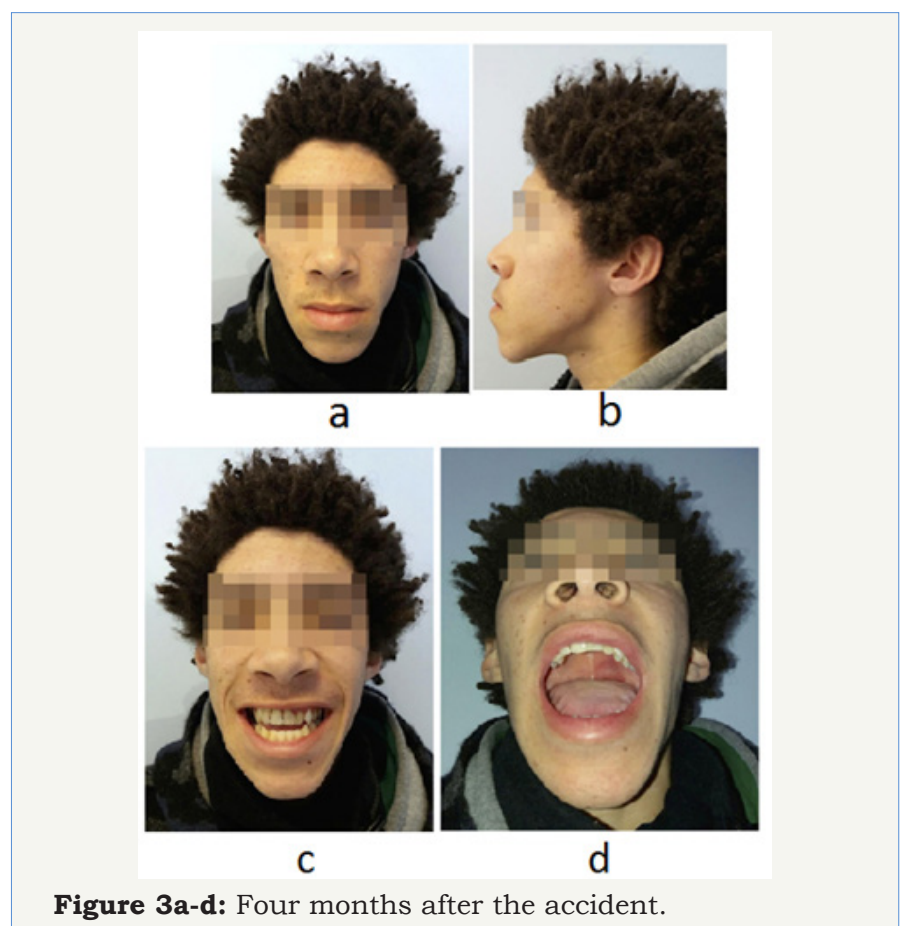

Figure 3a-d: Four months after the accident.

\section{Discussion}

Facial trauma can assume complex forms closely related to the mechanism of injury. Midface orofacial trauma may result in potentially disfiguring injuries with important functional impact, as it commonly affects dental occlusion. The initial approach to complex facial trauma includes the confirmation of a patent airway, hemodynamic stability and hemorrhage control, along with a carefully collected history and complete physical examination. Imaging studies play an important role in the characterization of facial traumatic injuries, particularly in the presence of facial bone fractures. Plain radiography, despite being a simple and inexpensive exam, offers limited information in the setting of complex facial fractures. High-resolution CT is often the most valuable exam to diagnose and characterize facial fractures in the emergency department, and may also prove valuable to diagnose associated soft tissue injuries [1].

Principles of treatment in this type of injuries include exploration, debridement of devitalized tissue, generous wound irrigation and reduction of fractures achieving dental and bone stabilization. Although the maxilla is commonly involved in facial trauma, fractures of the hard palate are relatively infrequent and require high-energy trauma [2,3]. The management of maxillary and palatal fractures requires achievement of dental stability and previous occlusion as well as restoration of height and projection of the midface.

In the case we present, a metal wire caused a direct fracture and associated soft tissue lacerations through the alveolar process of the maxilla and palate in a oblique fashion, with associated horizontally-oriented fractures of the nasal floor and anterior wall of the left maxillary sinus. A small area of anterior vestibular mucosa was the only tissue providing vascularization to the fragment and the preservation of this mucosa avoided its complete avulsion. According to a 2016 systematic review by Moss et al. [2] of 310 patients with hard palate fractures, sagittal or Type I fractures account for the vast majority of the fractures described in the literature (89\%), while transverse or Type II fractures, as in the one we describe, are the least frequent of the three types, accounting for only $5 \%$ of the cases, with comminuted or Type III fractures accounting for the remaining $6 \%$ of the cases [2]. Palatine fractures are most frequently managed with plate fixation of the alveolar ridge associated with either closed reduction of the palatal vault with circumdental wiring or open reduction with internal plate fixation [2]. More recently, external fixation [2] techniques have been described $[4,5]$.

In the particular case we present, surgical treatment of fractures with microplate osteosynthesis of the anterior maxilla and alveolar ridge was made difficult by the approach through the existent lacerations in the lateral vestibular mucosa, since an anterior vestibular approach would compromise the vascularization of the fractured bone fragment. The palate fracture was also addressed with internal microplate fixation of the palatal vault. Additionally, immobilization of the upper dental arch with Erich arch bar fixation and steel wire dental cerclage was performed for increased dental 
stability. Complications were minor and did not require additional intervention. The administration of antibiotic prophylaxis, including early prophylaxis and a postoperative antibiotic course, is recommended in cases of open fractures such as this for prevention of postoperative infections [6]. Prescription of liquid and soft diet in the first post operatory days allows favorable wound healing. After surgical reduction of the fracture, the patient was confirmed to have a class III skeletal and dental malocclusion pattern previously to the accident. The patient confirmed that the treatment applied was effective in restoring previous dental occlusion. There were no long-term functional or esthetic consequences of the suffered trauma.

\section{Conclusion}

Facial trauma can be complex, associating soft tissue injuries with bone fractures in sometimes rare patterns. The management of such complex injuries often demands an individualized approach. Careful diagnosis using adequate imaging studies, adequate preoperative planning, and proper wound care and infection prevention are common principles important to the successful treatment of these patients.

\section{References}

1. Cooper PW, Kassel EE, Gruss JS (1983) High-resolution CT scanning of facial trauma. AJNR Am J Neuroradiol 4(3): 495-498.

2. Moss WJ, Kedarisetty S, Jafari A, Schaerer DE, Husseman JW (2016) A review of hard palate fracture repair techniques. J Oral Maxillofac Surg 74(2): 328-336.

3. Ferreira PC, Barbosa J, Braga JM, Rodrigues A, Silva AC, et al. (2016) Pediatric facial fractures: A Review of 2071 Fractures. Ann Plast Surg 77(1): 54-60.

4. Cienfuegos R, Sierra E, Ortiz B, Fernández G (2010) Treatment of palatal fractures by osteosynthesis with $2.0-\mathrm{mm}$ locking plates as external fixator. Craniomaxillofac Trauma Reconstr 3(4): 223-230.

5. Ma D, Guo X, Yao H, Chen J (2015) Transpalatal screw traction: A simple technique for the management of sagittal fractures of the maxilla and palate. Int J Oral Maxillofac Surg 43(12): 1465-1467.

6. Abubaker AO (2009) Use of prophylactic antibiotics in preventing infection of traumatic injuries. Dent Clin North Am 53(4): 707-715.
Creative Commons Attribution 4.0

International License

For possible submissions Click Here

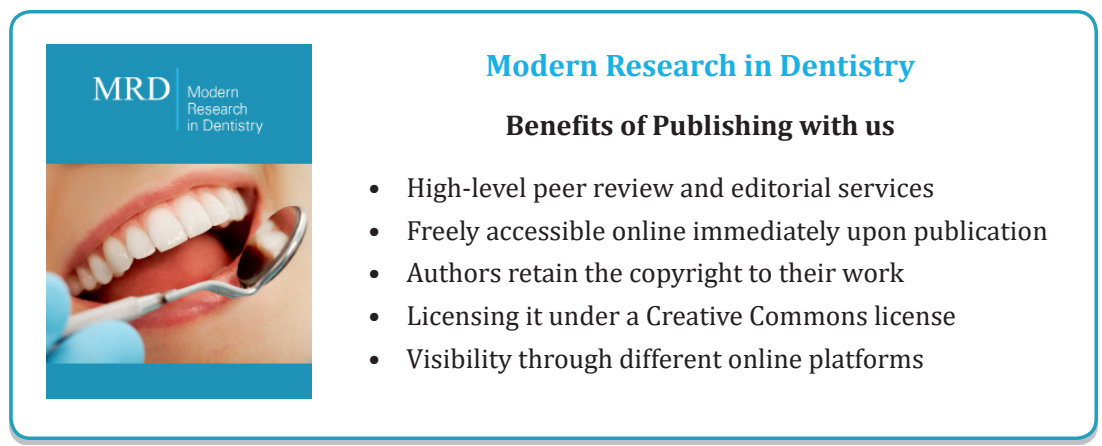

